\title{
LAS TRES ECONOMÍAS DEL SECTOR ARTÍSTICO
}

\author{
Izaskun Echevarría Madinabeitia \\ Universidad de Zaragoza. Dpto. Expresión Musical, Plástica y Corporal
}

\section{Resumen}

Gracias a las reflexiones desarrolladas a partir de la obra History Zero de Stefanos Tsivopoulos mostrada en la Bienal de Venecia de 2013, en relación con el análisis de Bourdieu acerca del sistema del arte, nos adentramos en las ambigüedades de conceptos tales como valor y precio para entender que lo que obtenemos de nuestra labor es la consecuencia de monetizar nuestra fuerza de trabajo dentro de un sistema mercantil. Este es un aspecto que debemos aprender a manejar, ya que no es algo dado dentro de un marco perfectamente regulado, sino una potencia de la que extraer rentabilidad, un espacio donde se establecen relaciones mutantes entre agentes diversos que atraviesan vida y praxis artística. A partir del propio lenguaje del arte, así como de la investigación en arte y la gestión cultural, tocando aspectos del derecho y la economía, este artículo reflexiona acerca de la relación entre arte y trabajo.

\section{Palabras clave: ECONOMÍA DEL ARTE; CRISIS ECONÓMICA; SISTEMA DEL ARTE; SOCIOLOGÍA DEL ARTE; CAPITALISMO}

\section{THE THREE ECONOMIES OF THE ARTISTIC SECTOR}

\section{Abstract}

Thanks to the analysis developed from the work History Zero Stefanos Tsivopoulos shown at the Venice Biennale 2013, in connection with the analysis of Bourdieu about the system of art, we delve into the ambiguities of concepts such as value and price to understand that what we get from our work is the result of monetizing our work force within a mercantile system. This is an aspect that we must learn to handle, since it is not something given within a perfectly regulated framework, but a power from which to extract profitability, a space where mutant relationships are established between diverse agents who go through life and artistic praxis. From the language of art itself, as well as art research and cultural management, touching on aspects of law and Economics, the article reflects on the relationship between art and work

\section{Keywords: ART ECONOMICS; ECONOMIC CRISIS; ART SYSTEM; ART SOCIOLOGY; CAPITALISM}

\footnotetext{
Echevarría Madinabeitia, Izaskun. 2019. "Las tres economías del sector artístico". AusArt 7(2): 153-166. D0I: 10.1387/ausart.21152
}

\section{AUSART}




\section{INTRODUCCIÓN}

A raíz de la crisis económica iniciada en 2008, el desmantelamiento del sistema de producción artística institucional — vinculado a salas de exposiciones municipales y centros de arte-generó un panorama desolador en el sistema del arte. No sólo reclamar el pago de honorarios para los productores de exposiciones parecía cada vez más impropio, sino que buena parte de los demás profesionales para la producción de exposiciones como montadores, traductores o diseñadores gráficos habían desaparecido tras la limpieza de gasto público que los ayuntamientos aplicaron ante las políticas de recortes.

Esta situación provocó que en muchas salas de exposiciones municipales toda la producción de la exposición, desde el montaje hasta el diseño de la publicación, recayese en la única figura del artista, abandonado a su suerte. Sin un presupuesto para la producción, además de no poder contar con los demás profesionales, muchos de estos espacios se vieron sin contenidos o bien, con contenidos en los que el doityourself era el modus operandi de un sector raquítico que tendía al amateurismo.

Al ver sus capacidades tremendamente limitadas para la producción en sala o espacialización de proyectos, los artistas - y en general todos los productores culturales - se vieron obligados a repensar sus metodologías. Sin saber cómo trabajar en un espacio diseñado bajo las premisas estéticas del cubo blanco, se enfrentaban con un cubo más que blanco, vacío. Y ante este panorama, muchos dieron la espalda a las instituciones públicas para crear sus propios espacios fuera de ellas. Este fenómeno que ya sucedió en los noventa ha ido generando numerosos estudios, publicaciones y obras conscientes de la importancia de analizar las sucesivas crisis del capitalismo.

En el campo de la práctica artística contamos con numerosos proyectos cuyos discursos se enmarcan en la crítica institucional o en un análisis crítico sobre la economía actual. Partiendo de presupuestos raquíticos o nulos, muchos de los proyectos contemporáneos en el ámbito estatal, en esta última década, se decantaron por la autofinanciación, aunque con el claro propósito de dejar de manifiesto las contradicciones del propio sistema del arte.

Lo cierto es que los artistas seguimos produciendo y es desde esta sensación de agotamiento casi de extenuación, desde la que nos preguntamos: ¿qué nos conduce a los trabajadores de la cultura a llegar a esta situación paradójica? Es decir, ¿qué hace que estemos constantemente produciendo si la mayor 
parte de las veces no recibimos remuneración económica alguna a cambio? ¿En qué consiste la recompensa de esta especie de castigo autoinflingido? Y, ¿por qué nos autoexplotamos si en este sistema económico en el que vivimos tenemos tan pocas opciones de rentabilizar nuestro trabajo?

\section{ECONOMÍA INFORMAL, SIMBÓLICA Y MONETARIA}

Es una realidad que la economía monetaria no constituye sino una parte de la cadena de valor que genera nuestra actividad tanto profesional como no profesional. En este estadio de relaciones, las prácticas artísticas entran a formar parte de un sistema mucho más complejo de lo que en un primer momento pareciera. El hecho artístico queda determinado por las relaciones humanas que nos constituyen y su valor por las interdependencias entre los distintos agentes que intervienen en la experiencia que se produce; porque, en primer lugar, lo que el arte produce son experiencias, antes que productos medibles, comunicables, transferibles e intercambiables.

Si tomamos como referencia el pensamiento de Bourdieu (1995), encontramos conceptos fundamentales acerca del tipo de economías que se establecen dentro del sistema del arte, tal y como lo conocemos hoy. Hablar de campo referido al sistema en el que operan los artistas y capital social, como la riqueza derivada de las relaciones internas que se establecen en ese campo, da cuenta de un territorio específico en contraposición al resto de la sociedad, en la que el habitus determina unos esquemas generativos comunes a partir de los que unos sujetos perciben el mundo y actúan en él.

Según el análisis del autor, existe un desprecio manifiesto por el mundo burgués en la época de Flaubert y Baudelaire. Un desprecio que sigue manifestándose en la actualidad por un sector significativo del campo del arte. En sus propias palabras, esos nuevos ricos sin cultura que demandaban y siguen alimentando hoy día un mercado de obras basadas en un gusto marcado por la impronta de la falsedad y la adulteración es lo que los artistas de la segunda mitad del S.XIX rechazaron categóricamente para construir un sistema propio en contraposición al "materialismo vulgar de los dueños de la economía (Bourdieu 1995, 95)". 
De hecho, este posicionamiento o lo que es lo mismo, la ruptura ética será considerada una dimensión fundamental en todas las rupturas estéticas durante la conquista de la autonomía (Bourdieu, 1995). En este campo que se instaura, "aquellos que pretenden ocupar en él posiciones dominantes se sentirán obligados a manifestar su independencia respecto a los poderes externos, políticos o económicos" (Bourdieu, 1995, 99).

El objetivo de esta ruptura fue posicionarse al margen de los requerimientos del mercado, manteniendo así una autonomía del campo artístico con respecto a otros agentes, lo que supuso crear un campo específico que abasteciera mutuamente a sus agentes. Mantener esta autonomía del arte suponía preservar la libertad en los discursos y las formas, independientemente de los requerimientos de la sociedad de la época. De esta manera, se establecía el giro que los artistas efectuaron en torno a su propia producción, independientemente de su contexto, momento en el cual se formuló la obra pura.

Este giro ha provocado interesantes contradicciones aún hoy día. Sabemos, por ejemplo, que la principal fuente de financiación de los artistas contemporáneos viene de los presupuestos de los fondos administrados por las instituciones públicas. De manera que no podemos perder de vista el sistema económico en el que nos encontramos inmersos. Según Bishop $(2016,13)$ "hasta inicios de los 90, el arte basado en la comunidad estuvo confinado a la periferia del mundo del arte; hoy se ha convertido en un género por derecho propio (...) pero florece más intensamente en países europeos con fuerte tradición de financiamiento público en las artes".

Quizá deberíamos conocer el momento previo al capitalismo si queremos entender otras formas de economía y de relación social que funcionan dentro del sistema del arte. El análisis que Silvia Federici realiza desde un enfoque feminista en Calibán y la bruja (2010) es muy recomendable. Según un pormenorizado y riguroso estudio histórico centrado en la Edad Media, expone que la modernidad y el capitalismo comienzan cuatro siglos atrás, momento en el que se despoja a las mujeres de su autonomía social y monetaria. "La persecución de brujas, tanto en Europa como en el Nuevo Mundo, fue tan importante para el desarrollo del capitalismo como la colonización y como la expropiación del campesinado europeo de sus tierras" (Federici 2015, 27).

Si desarrollamos los paralelismos que se establecen entre conceptos como lo productivo y lo improductivo o entre lo científico, instaurado a partir del conocimiento enciclopédico, y lo no científico, como herencia de la sabiduría popular, 
que Federici (2015) analiza como un conocimiento extirpado de las sociedades modernas, obtendremos dos vectores de fuerza que conviven en la sociedad actual. Según Federici a partir del cambio al capitalismo la reproducción de la fuerza de trabajo queda disociada y dejada al margen de la lógica de los Estados, concebidos ya como grandes empresas cuyos valores se basan en la productividad y la competitividad.

La crisis económica de la última década ha dejado de manifiesto que el capitalismo es un sistema basado en la desigualdad y que sus crisis son sistémicas. Si observamos el sistema capitalista en un contexto de crisis, vemos que se han puesto de manifiesto las patologías sociales que se mantenían en la base (Cabo de la Vega 2012). En su análisis crítico acerca del constitucionalismo social, Cabo de la Vega establece cinco limitaciones fundamentales que presentan patologías sistémicas. En el ámbito de lo social distingue siete patologías: el trabajismo (u obrerismo), el patriarcalismo, la estigmatización, el ugly sister competition, la trampa de la pobreza, el clientelismo, la autoprogramación, la ineficacia del gasto y el estatismo.

Los derechos sociales contribuyeron al ensalzamiento del trabajo formal (obrero) a costa de la desvalorización de otras formas de trabajo (reproductivo, de cuidado, de la economía informal) con la consiguiente degradación de los grupos de individuos que los ejercían. Sólo el trabajo a tiempo completo en el mercado formal, ejercido de forma continuada se consideraba digno de aprecio. (...) El mercado de trabajo de la posguerra se construyó con un patrón netamente patriarcal en el que las mujeres son desplazadas de sus puestos en la economía de guerra hacia los márgenes o el exterior del sistema. (...) Conforme la situación económica se deteriora (...) el grupo de trabajadores sanos con trabajo tiende a conceptualizar a los receptores como parásitos del sistema.

(Cabo de la Vega 2012, 38)

\section{'HISTORY ZERO' DE STEFANOS TSIVOPOULOS}

El colonialismo que llevó a los europeos a escrutar todos los rincones del planeta en busca de mano de obra y materias primas baratas fue - en particular durante el último tercio del s.XIX - en busca, también, de nuevos mercados donde dar salida a sus excedentes de producción. Es en esta época cuando 
surgen las grandes exposiciones universales como motor económico y estímulo al consumo, pero al mismo tiempo como tentativa para mostrar a los trabajadores europeos cómo era el mundo; ese mundo que ellos mismos estaban construyendo con su esfuerzo como actores de la revolución industrial (Canogar 1992).

El interés por el conocimiento y la cultura, así como muchos museos surgen en esta misma época como forma de entender enraizada en el conocimiento enciclopédico, es decir, en el estudio y la clasificación de los objetos.

La 55ª Bienal de Venecia, celebrada en 2013 se tituló II Palazzo Enciclopedico. Este título estaba inspirado en la obra del artista autodidacta italo-americano Marino Auriti, que en 1955 registró su diseño del Palazzo Enciclopedico en la oficina de patentes de Estados Unidos. El proyecto era un museo imaginario cuya función sería albergar el conocimiento acumulado en todo el mundo, conteniendo los mayores descubrimientos de la especie humana, desde la rueda hasta el satélite (Gioni 2013).

El propio comisario de la Bienal, Massimiliano Gioni $(2013,18)$, resumía la muestra aludiendo a "esas cosmologías personales — con su delirio omnisciente-como un fenómeno que arrojaba luz sobre la constante de reconciliar el sujeto con el universo, lo subjetivo con lo colectivo, lo específico con lo general y lo individual con la cultura de su tiempo".

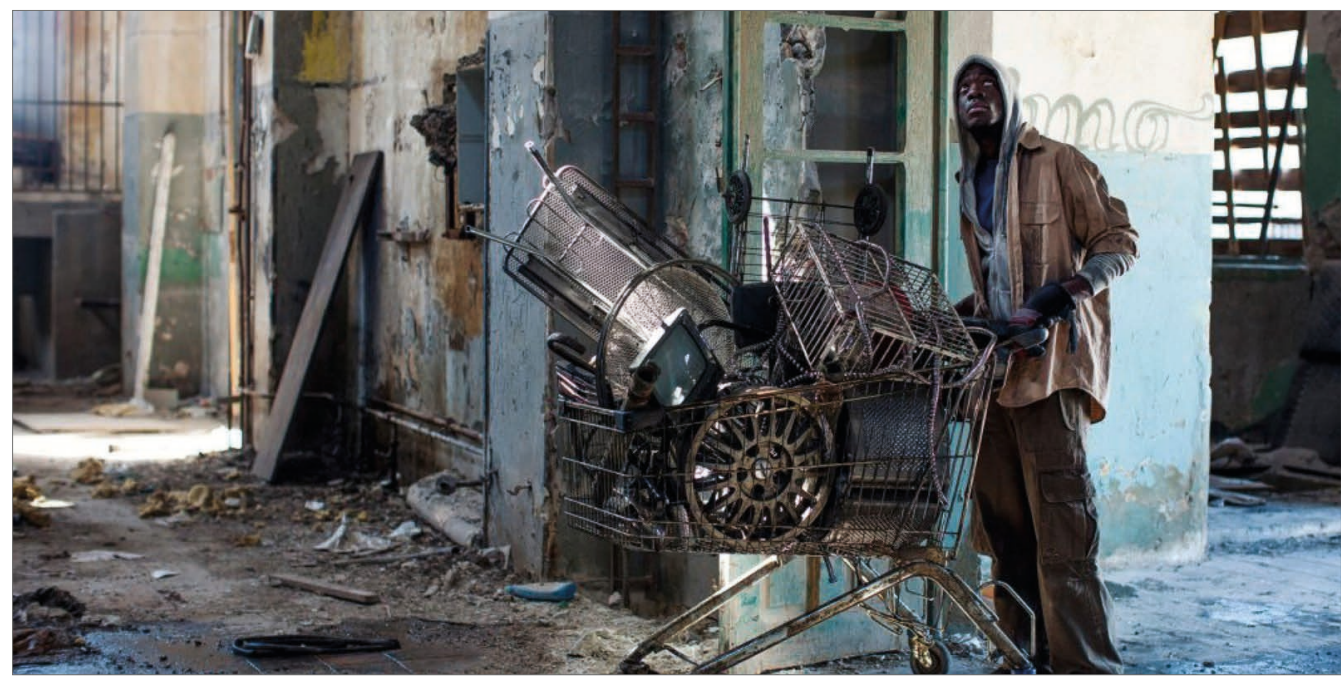

Imagen 1. Imagen tomada de "History Zero" de Stefanos Stivopoulos.

Fuente: The Greek Foundation, 2013 
En la $55^{a}$ Bienal de Venecia, el Pabellón de Grecia mostraba la obra de Stefanos Tsivopoulos, History Zero. La parte preliminar ubicada en una sala lateral del pabellón estaba compuesta por una serie de 32 ejemplos de monedas alternativas de todo el mundo, algunas de ellas modelos históricos que ejemplificaban las maneras en las que algunas comunidades habían desarrollado formas organizativas propias basadas en el intercambio monetario.

En el interior del pabellón se encontraba una videoinstalación con tres pantallas dispuestas formando un semicírculo. Las tres escenas de las que se componía la obra se desarrollaban en Atenas y nos hablaban del valor de las cosas, de la fluctuación constante de su valor y de lo anecdótico del valor del dinero. Al estar dispuestas de manera simultánea, podía apreciarse que los tres relatos se complementaban entre sí.

La primera estaba protagonizada por una coleccionista de arte que vivía en una gran mansión rodeada de lujo mientras se dedicaba a crear flores con papiroflexia con billetes de euro. La segunda escena retrataba el periplo de un artista conceptual, vestido de negro, que aterrizaba en Atenas con intención de producir una obra para lo cual deambulaba por la ciudad mientras grababa escenas callejeras cotidianas durante horas. La última mostraba el relato de un inmigrante africano que recogía chatarra fascinado por las formas de los objetos que encontraba, desde que se ponía el sol hasta la madrugada.

En un momento, durante la búsqueda que iniciaban los tres personajes, sus relatos se entrelazaban y la obra cobraba sentido; se convertía en un círculo virtuoso. Así, History Zero mostraba dónde radicaba el valor de las cosas para cada personaje, porque lo que para uno era basura para otro podía ser lo más valioso, aquello que buscaba en el mundo.

Volvamos al plano del sistema del arte, tal y como lo describe Pascal Gielen (2014) a partir de Bourdieu. El autor nos presenta la figura de Jan Hoet, que constituye un valioso ejemplo para entender la articulación de los tres tipos de economías que venimos introduciendo. Hoet construyó el prestigioso museo de Gante. Para su conformación utilizó lo que denominó una espiral acumulativa gracias a la que consiguió conformar una colección de gran valor en la que operaban los tres tipos de economías o niveles de valor derivados de las obras de arte.

En primer lugar, la economía informal, basada en "la confianza y la amistad, el reconocimiento mutuo y el respeto. Dentro de éste, el intercambio basado en 
el principio de reciprocidad se encuentra dentro de las posibilidades" (Gielen 2014, 71). Este tipo de economía genera una gran expectativa; la confianza entre iguales genera un potencial, una promesa y eso atrae a otras personas que se acercan ante lo que parece ser una buena apuesta a futuro. Es lo que a todas luces hoy día estamos acostumbrados a designar como una colaboración. Podría ser lo que el artista conceptual retrata en Atenas, en la obra de Tsivopoulos.

En segundo lugar, nos plantea la economía simbólica como aquello que puede operar en la distancia a favor del artista y también como aquel elemento separable en el tiempo. Es decir, "si una obra tiene una gran cantidad de capital simbólico, puede ser utilizado para adquirir otras obras con un valor simbólico importante" (Gielen 2014, 71). Evidentemente, este estadio depende directamente de su contexto, pero tanto los billetes trasformados como la chatarra en otro orden pueden funcionar como ejemplos.

Y, en tercer lugar, la economía monetaria, siempre pensando en el ejemplo que utiliza Pascal Gielen. Cuando no operan ninguna de las otras dos actúa la transacción monetaria para conseguir un bien o un servicio, aunque puntualiza: "lo interesante aquí es la interacción que se produce entre estas economías informales, simbólicas y monetarias" (id.).

Según Gielen es posible pensar en estos tres tipos de economía que operan en el ámbito artístico. La economía del arte es un compendio de economías que va mucho más allá del mercado. Estas economías, cuyos vértices interdependientes plantearían tres cadenas de valor interconectadas y elásticas, a modo de triángulo, conformarían hipotéticas polifonías con las que podríamos crear infinitos patrones relacionales.

El esquema fijo de la Imagen 2 representaría un solo momento de un triángulo dinámico en el que, si estirásemos de uno de sus vértices descentraríamos la proporción de la figura, lo que resultaría en asignar más valor a un aspecto frente al otro dentro de una lógica de valor interconectada en un todo indisoluble.

Debemos puntualizar aquí que el concepto economía informal es peligroso incluso desde la consideración de su propio creador Keith Hart, ya que puede llegar a naturalizar formas de discriminación o servir para implementar modelos asistenciales o neoliberales al sustituir la inversión pública en esos modelos por este tipo de economía. De hecho, una de las batallas internas de las 
asociaciones profesionales que no alcanzan a tener suficiente capital como para pagar a los miembros que conforman los equipos de trabajo y desempeñan tareas propias de puestos de trabajo especializado no remunerado, es si es legítimo pagarles y convertir su labor abiertamente en un trabajo. Esto supone una absoluta contradicción teniendo en cuenta la precariedad económica en la que se encuentran muchos de estos artistas que desarrollan su labor dentro de unas estructuras asociativas cuyo ánimo es mejorar el campo artístico de manera global.

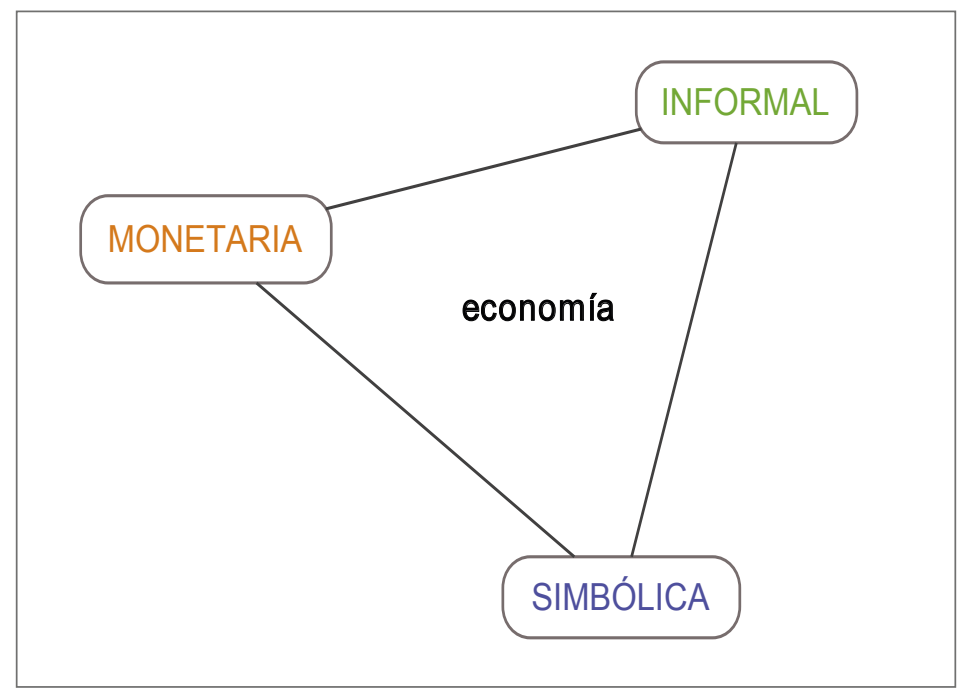

Imagen 2. Triángulo de las economías.

Fuente: Elaboración propia, 2017

De alguna manera, efectivamente, esta economía informal tiende a normalizar la pobreza considerando heroica la labor titánica de estos maltrechos sujetos que ven cómo su situación económica personal se hace insostenible en el largo plazo. Es importante recordar que sólo pueden sostenerse en el tiempo ocupando su energía con trabajo no remunerado aquellas personas que disfrutan de un bienestar económico a priori, lo cual convierte al arte en una actividad donde el éxito o el fracaso dependen de cuestiones de clase.

Sin embargo, como nos recuerdan en su estudio Marta Pérez Ibáñez e Isidro López-Aparicio, lo que destaca de esta actividad y de este campo es "la capacidad de resiliencia de los artistas ante los cambios de los contextos como el hecho de que son los artistas y su entorno social los principales mecenas 
del arte, los que aportan un mayor capital en la existencia del mundo del arte contemporáneo en España" $(2017,12)$.

\section{ALGUNAS CONCLUSIONES: LA \\ PROFESIONALIZACIÓN DEL ARTE COMO PROBLEMA}

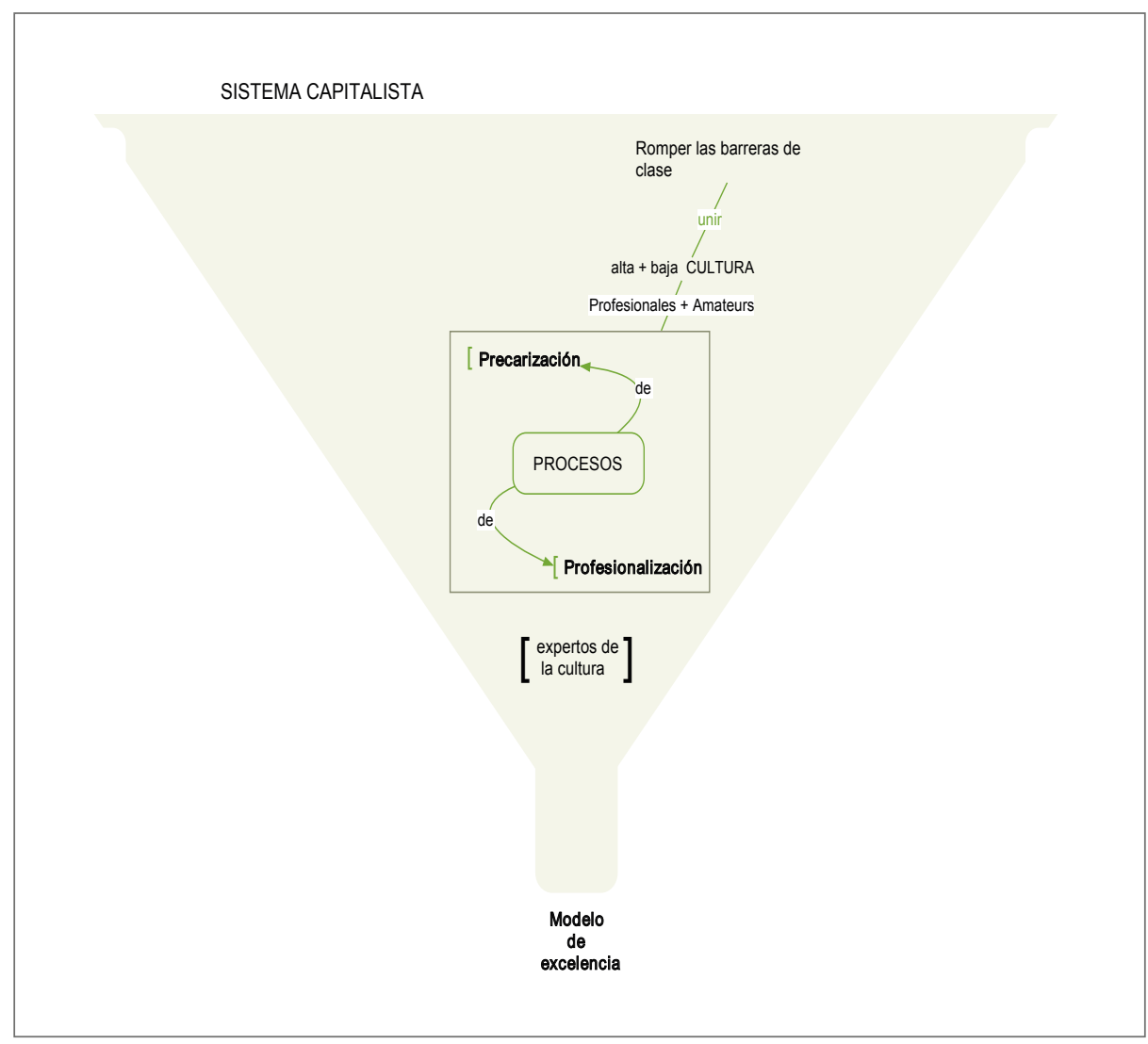

Imagen 3. La ley del embudo.

Fuente: Elaboración propia. 2018

A partir de este esquema podemos ver dos procesos que coexisten actualmente en políticas culturales: uno en el que se propone romper las barreras de clase para unir alta y baja cultura (proceso de precarización) basado en la 
cultura como lo común; y otro en el que los expertos en cultura producen bienes que son ofrecidos para su consumo por parte de la ciudadanía (proceso de profesionalización) dentro de lo que viene siendo un modelo de excelencia.

Ambos modelos generan sus problemas propios, tanto para la existencia de una cultura sin barreras de clase y de calidad, como para la existencia de un sector con profesionales bien remunerados que gocen de un reconocimiento social, atendiendo al sistema capitalista en el que nos encontramos.

Hay que señalar como primer problema que el sistema capitalista aplica su propia lógica de mercado sobre la cultura como producto, y que existe una tendencia infinita al adelgazamiento de las instituciones públicas. En segundo lugar, y desde la perspectiva del arte, que ese producto es muchas veces el propio sujeto. Maite Aldaz $(2017,17)$ nos recuerda en este sentido que "la producción de la subjetividad significa, en cualquier caso, no sólo que la subjetividad es producida, sino también que es productiva" porque escapa al control originario del capital que la crea para nutrirse a través de ella y "desborda esa función produciendo otras formas de socialidad disidentes" (Read 2003 citado en Aldaz 2017, 17). Encontramos infinidad de proposiciones artísticas que trabajan en este sentido. Es el caso de la obra Afrodita de Nuria Güell.

En septiembre de 2017, Nuria Güell desarrollaba su trabajo titulado Afrodita como parte de la exposición 'Arcana Imperii. Investigaciones en Burocracia' curada por Oriol Fontdevila que tuvo lugar en el Centre de Cultura Contemporània El Carmen de Valencia. La obra exponía a la institución, mediante rueda de prensa, la necesidad de cotizar las cuotas mínimas a la seguridad social como autónoma para poder disfrutar así de un permiso de maternidad e instaba a quien financiaba el proyecto a que fueran pagadas como parte de la producción de obra.

Con gran acierto, la artista ponía en evidencia la sangrante elección a la que muchas mujeres del sistema del arte se enfrentan: un sistema de becas y subvenciones que fomenta la inestabilidad, ya que su éxito es momentáneo y muchas veces intencionadamente aislado. Esta precariedad laboral (si nos atrevemos a llamar trabajo a la actividad artística) es parte de las problemáticas vinculadas a la profesionalización del arte, que en el caso de las mujeres que desean ser madres existe como disyuntiva a tener que elegir entre la maternidad o una carrera profesional precaria. 


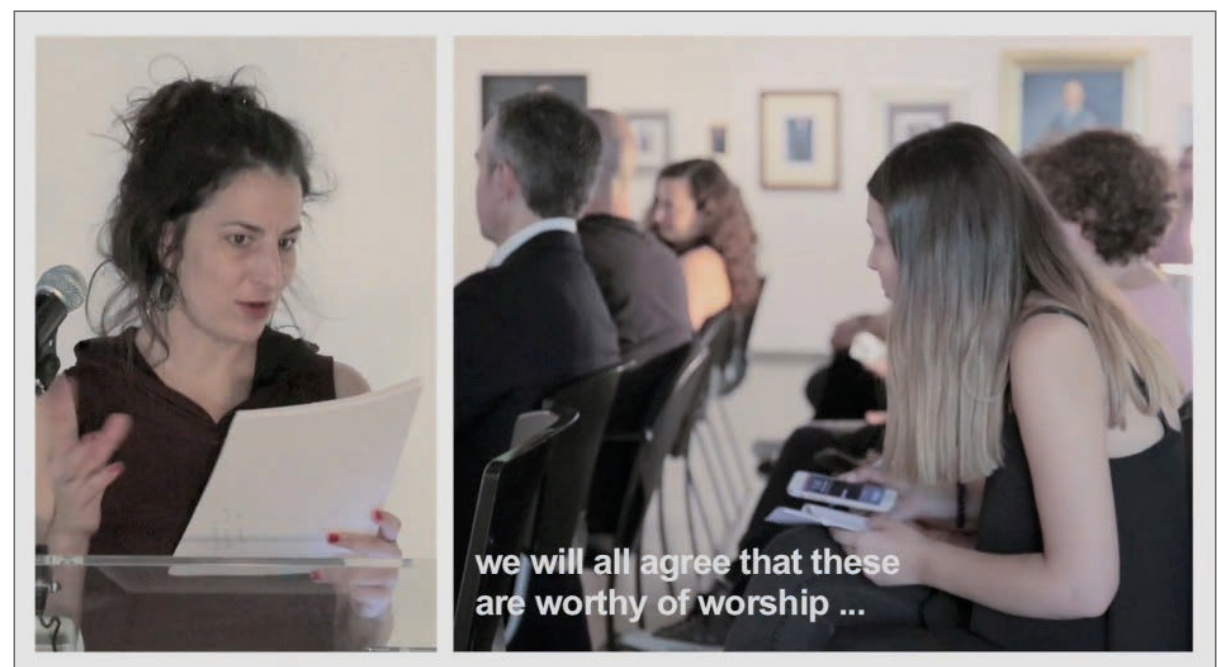

Imagen 4. Fotograma de la performance Afrodita de Nuria Güell.

Fuente: web de la artista

El reconocimiento de la remuneración del/la artista en forma de honorarios planteada hoy día como superación de la comercialización de artefactos como única vía de financiación sigue suscitando controversia dentro de muchas instituciones que financian el arte contemporáneo. De hecho, en términos absolutos, la tendencia es más bien la contraria. Sin embargo, la realidad es que una vez desvinculadas de su autor/a, las obras toman caminos que escapan a su control, entrando muchas veces en contradicciones ontológicas entre los motivos de su génesis y los efectos de su tránsito material por el mundo.

La lucha por la regulación del sistema del arte y la cultura ya sea desde la producción artística, la gestión cultural, la investigación en arte o el derecho es un campo que ha vuelto a disfrutar de una gran atención en estos últimos años de crisis económica. De hecho, la aprobación por unanimidad del Estatuto del artista en junio de 2018 es una muestra más de esta atención a la precariedad laboral del sector cultural, siendo una demanda que viene desde hace décadas. En cualquier caso, la propuesta de solución requiere de un largo proceso que afecta a los Ministerios de Trabajo y Hacienda para la creación de un régimen especial para los trabajadores de la cultura atendiendo a su condición de intermitencia y, por tanto, de excepcionalidad dentro de las leyes que regulan las actividades económicas y el sistema de cotizaciones a la seguridad social.

Conocer la existencia de organismos autónomos, no-institucionales, y participar en ellos debería ser uno de los objetivos de cualquier trabajador que como 
tal se precie. Por ello, cabe citar el desempeño de las asociaciones de artistas visuales a nivel estatal para permitir la creación de un marco de regulación y acceso a los recursos públicos disponibles mediante sistemas transparentes. Su batalla por defender los derechos de los artistas en España es de suma importancia durante la última década. Uno de los documentos más recurrentes es el Manual de Buenas Prácticas editado por la Unión de Artistas Contemporáneos (Brun 2008).

En contextos que escapan a la lógica institucional, conocer la existencia de una Normativa para la creación de jurados y comisiones técnicas, así como la Propuesta para la regulación de convocatorias de AVVAC (Asociación de Artistas Visuales de Valencia, Alacant i Castelló) puede convertirse en una hoja de ruta ineludible que exponer a gestores e instituciones públicas que administran los recursos públicos.

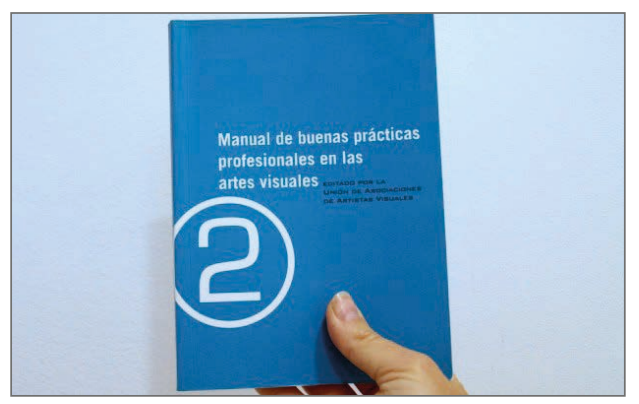

Imagen 5. Manual de Buenas Prácticas. Fuente: web de La Unión de Artistas Contemporáneos.

Nos queda claro que la recompensa que obtenemos del mundo del arte es, en un alto grado, de tipo emocional. Eso explicaría por qué somos nosotros mismos los que nos nutrimos del sistema y, por tanto, nos esforzamos por mantenerlo y alimentarlo como lugar esencial para nuestro desarrollo vital. Es esa especie de precariado rabioso que tira hacia adelante a pesar de no conseguir beneficio económico que permita una sostenibilidad suficiente, e incluso a veces, apenas reconocimiento social. El arte nos construye como sujetos y nos posibilita una serie de potencias que usar en el día a día, aunque rentabilizar nuestra actividad siga teniendo que pasar por la mutación constante como condición de su operatividad.

\section{Referencias bibliográficas}

Aldaz Enrique, Maite. 2017. “Institución Arte y Precariedad: Las prácticas artísticas en la época de la mercantilización de la vida". En Producción artística en tiempos del precariado laboral, edición a cargo de Carmen Navarrete \& Juan Vicente Aliaga, 13-39. Madrid: Tierra de Nadie

Aramburu Gil, Nekane, dir. 2011. Historia y situación actual de los colectivos de artistas y espacios independientes en el estado español (1980-2010): La otra historia. Vitoria-Gasteiz: N. Aramburu 
AVVAC Artistes Visuals de València, Alacant i Castelló. 2016a. "Normativa para la creación de jurados y comisiones técnicas". Avvac.net, 11 dic. http://www.avvac.net/normativa-para-la-creacion-de-jurados-y-comisiones-tecnicas/

AVVAC Artistes Visuals de València, Alacant i Castelló. 2016b. "Propuesta para la regulación de convocatorias". Avvac.net, 11 dic. http://www.avvac.net/propuesta-para-la-regulacion-de-convocatorias/

Bishop, Claire. 2017. Infiernos artificiales: Arte participativo y políticas de la espectaduría. Traducción Israel Galina Vaca. Ciudad de México: Taller de Ediciones Económicas

Bourdieu, Pierre. 1995. Las reglas del arte: Génesis y estructura del campo literario. Traducción de Thomas Kauf. Barcelona: Anagrama

Brun Menéndez, Lluís. 2008. Manual de Buenas prácticas profesionales en las artes visuales. Madrid: Unión de Asociaciones de Artistas Visuales. https://avvac.files.wordpress. com/2013/05/avvac_manual_buenas_practicas_02.pdf

Cabo de la Vega, Antonio de. 2012. "El fracaso del constitucionalismo social y la necesidad de un nuevo constitucionalismo". En Por una asamblea constituyente: Una solución democrática a la crisis, Marco Aparicio Wilhelmi et al., 29-48. Madrid: Sequitur. https://www.ucm.es/ data/cont/docs/603-2013-12-19-asambleaconstituyente.pdf

Canogar, Daniel. 1992. Ciudades efímeras: Exposiciones universales; Espectáculo y tecnología. Madrid: Julio Ollero

Echevarria Madinabeitia, Izaskun. 2019. "De la crítica institucional a las instituciones críticas: Políticas culturales y autogestión en arte en el contexto español, 2007-2017". Tesis Univ. Politècnica de València. http://hdl.handle.net/10251/122314

Federici, Silvia. 2015. Calibán y la bruja: Mujeres, cuerpo y acumulación primitiva. Traducción, Verónica Hendel \& Leopoldo Sebastián Touza. Madrid: Traficantes de Sueños

Fontdevila Subirana, Oriol. 2013. "Arqueología preventiva". Ciclo de exposiciones en el Espai 13 de la Fundació Joan Miró. http://oriolfontdevila.net/es/arqueologia-preventiva/

Fontdevila Subirana, Oriol. 2017. El arte de la mediación. Bilbao: Consonni

Gielen, Pascal. 2014. El murmullo de la multitud artística: Arte global, política y posfordismo. Traducción a cargo de Hugo López-Castrillo \& Alejandro Arozamena. Madrid : Brumaria

Gioni, Massimiliano, ed. 2013. Il palazzo enciclopedico: Guida breve. Venezia: Marsilio

Güell, Nuria. 2017. "Afrodita”. Conf. Centre El Carme, Valéncia, 22 sept. Vídeo de Vimeo, 11:52 min. https://vimeo.com/238026459

Pérez Ibáñez, Marta \& López Aparicio, Isidro. 2018. La actividad económica de los/las artistas en España: Estudio y análisis. Coord., Ecosistema del Arte. Madrid: Fundación Antonio de Nebrija

Tsivopoulos, Stefanos. 2013. "Biennale Arte 2013-Greece". Intervista alla 55. Esposizione Internazionale d'Arte. Vídeo de Youtube, 3:03 min. https://www.youtube.com/watch?v=hDvlUqvxl3o

Tsivopoulos, Stefanos. 2014. "History Zero" Thegreekfoundation.com, 4 sept. www.thegreekfoundation.com/art/history-zero-stefanos-tsivopoulos

$$
\text { (Artículo recibido: 14-10-19; aceptado: 10-01-20) }
$$

